\title{
Revan Indices and Revan Polynomials of Silicon Carbide Graphs
}

\author{
Rehana Ashraf \\ Department of Mathematics \\ Lahore College for Women University, Jhang Campus Pakistan \\ Sadia Akhter \\ Department of Mathematics \\ Lahore College for Women University, Jhang Campus Pakistan
}

\begin{abstract}
Topological polynomials are algebraic expressions which are related to the topology of graphs up to graph isomorphism. They are used to indicate the invariants of graphs of chemical structures. In a chemical graph, vertices and edges correspond to atoms and bonds respectively. The quantitative structure property relationship (QSPR) depicts a connection between the structure and the properties of molecules. There are numerous types of topological indices among them degree based are more popular. In this paper we compute Revan first index, Revan second index, Revan third index and their polynomials for list of silicon carbide graphs. Comparison between Revan first index, Revan second index and Revan third index is also part of our study.
\end{abstract}

Keywords-Chemical graph theory; chemical graph; atoms; bonds; silicon carbide; Revan vertex degree; first Revan index; second Revan index, third Revan index, Revan polynomials.

\section{INTRODUCTION}

Chemical graph theory is a topology branch of mathematical chemistry which deals with applications of graph theory to mathematical structure of chemical phenomena. Molecular graphs are the basic models of chemical graph theory in which vertices corresponds to vertices and edges correspond to bonds. The quantitative structure property relationship (QSPR) depicts a connection between the structure and the properties of molecules [1], [2]. Various numerical graph invariants and their related polynomials have been defined and used for correlation analysis in theoretical chemistry, pharmacology, toxicology and environmental chemistry. A topological index is a type of molecular descriptor which is calculated for a molecular graph of chemical phenomena. Topological indices are used to study the topology and graph invariants of molecular graphs. For a simple, connected and undirected graph $G=(V(G), E(G))$ with vertex set $V(G)$ and edge set $E(G), u v$ is an edge with end vertices $u$ and $v, d_{u}$ denotes the ordinary degree of vertex $u$ in $G, \Delta(G)$ denotes the maximum degree of $G$ and $\delta(G)$ is the minimum degree of $G$. For a simple and connected graph $G$, V. R. Kulli [5] introduced Revan first index, Revan second index and third Revan index as:

$$
R_{1}(G)=\sum_{u v \in E(G)}\left(r_{u}+r_{v}\right)
$$

$$
\begin{aligned}
& R_{2}(G)=\sum_{u v \in E(G)}\left(r_{u} \cdot r_{v}\right) \\
& R_{3}(G)=\sum_{u v \in E(G)}\left|r_{u}-r_{v}\right|
\end{aligned}
$$

Where $r_{v}$ is Revan degree of a vertex $v$ defined by Kulli as $r_{v}=\Delta(G)+\delta(G)-d_{v}$.

In 2018, V. R. Kulli [6] introduced first, second and third Revan polynomials of a simple connected graph $G$ as:

$$
\begin{aligned}
& R_{1}(G, x)=\sum_{u v \in E(G)} x^{\left(r_{u}+r_{v}\right)} \\
& R_{2}(G, x)=\sum_{u v \in E(G)} x^{\left(r_{u} \cdot r_{v}\right)} \\
& R_{3}(G, x)=\sum_{u v \in E(G)} x^{\left|r_{u}-r_{v}\right|}
\end{aligned}
$$

\section{SILICON CARBIDE GRAPHS}

We consider a family of silicon carbide graphs, and calculate necessary computations in this section. This family includes eight silicon carbide graphs with the names and notation as: $S i_{2} C_{3}-I[n, m], S i_{2} C_{3}-I I[n, m], S i_{2} C_{3}-$ $I I I[n, m], S i C_{3}-I[n, m], S i C_{3}-I I[n, m], S i C_{3}-$

$I I I[n, m], \mathrm{SiC}_{4}-I[n, m], \quad$ and $\mathrm{SiC}_{4}-I I[n, m]$ for arbitrary $n, m \geq 1$. Here we list some graph terms and properties which is a common possession of all these eight graphs:

i.In all graphs of silicon carbides, silicon atoms $S i$ are colored blue and carbon atoms $C$ are colored red, green color edges are used to connect two cells in a colums and red color edges are used to connect two cells in a row.

ii.In all graphs, vertices have ordinary degree 1,2 or 3 and since $\Delta(G)+\delta(G)=4$, so vertices have Revan degree 3,2 or 1 respectively.

iii. An edge $u v$ has a type $\left\{r_{u}, r_{v}\right\}$ if vertex $u$ has Revan degree $r_{u}$ and vertex $v$ has Revan degree $r_{v}$. 
iv.Edge sets of all eight silicon carbide graphs are portioned with respect to type defined above and cardinalities of partite sets are computed.

Description and computations about these graphs are as follows:

1. The $2 D$ structure of silicon carbide graph $G \cong$ $S i_{2} C_{3}-I[n, m], n, m \geq 1$, is shown in Figure 1 .

2 .

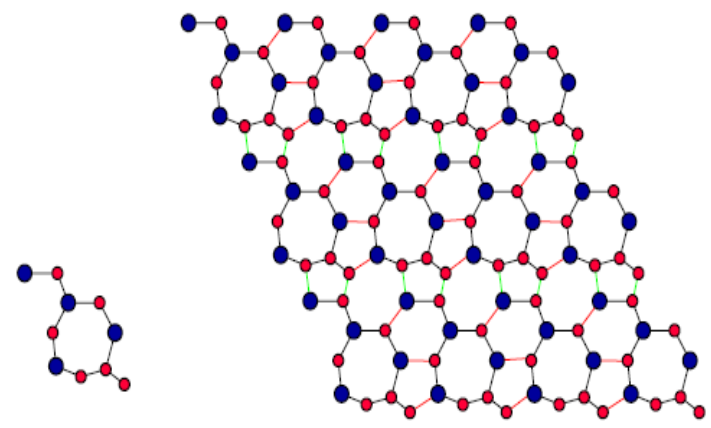

Figure 1

This structure consists of $n$ cells (in a row) and $m$ connected rows. Left figure is a unit cell and right figure is of $\mathrm{Si}_{2} \mathrm{C}_{3}-$ $I[4,3]$. Order of this graph is $10 \mathrm{~nm}$ and size is $15 \mathrm{~nm}-$ $2 n-3 m$. Edge partition and cardinalities of partite sets are given as:

$R E_{3,2}=\left\{u v \in E\left(S i_{2} C_{3}-I[n, m]\right) \mid r_{u}=3, r_{v}=2\right\}$,

$R E_{3,1}=\left\{u v \in E\left(S i_{2} C_{3}-I[n, m]\right) \mid r_{u}=3, r_{v}=1\right\}$,

$R E_{2,2}=\left\{u v \in E\left(S i_{2} C_{3}-I[n, m]\right) \mid r_{u}=2, r_{v}=2\right\}$,

$R E_{2,1}=\left\{u v \in E\left(S i_{2} C_{3}-I[n, m]\right) \mid r_{u}=2, r_{v}=1\right\}$,

$R E_{1,1}=\left\{u v \in E\left(S i_{2} C_{3}-I[n, m]\right) \mid r_{u}=1, r_{v}=1\right\}$,

And cardinalities are $\left|R E_{3,2}\right|=1,\left|R E_{3,1}\right|=1,\left|R E_{2,2}\right|=$ $n+2 m,\left|R E_{2,1}\right|=6 n+8 m-9,\left|R E_{1,1}\right|=15 m n-9 n-$ $13 m+7$.

3. The $2 D$ structure of $S i_{2} C_{3}-I I[n, m], n, m \geq$ 1 , is shown in Figure 2.

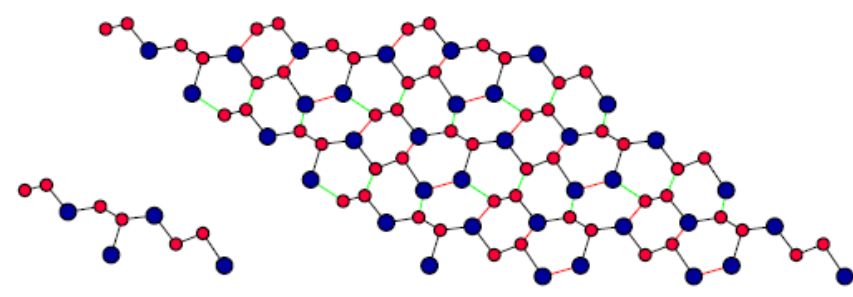

Figure 2

This structure consists of $n$ cells (in a row) and $m$ connected rows. Order of this graph is $10 \mathrm{~nm}$ and size is $15 \mathrm{~nm}-$ $3 n-3 m$. Left picture is a unit cell and right is of $S i_{2} C_{3}-$ $I I[3,3]$. Edge set is partitioned into following sets: $R E_{3,2}=\left\{u v \in E\left(S i_{2} C_{3}-I I[n, m]\right) \mid r_{u}=3, r_{v}=2\right\}$, $R E_{3,1}=\left\{u v \in E\left(S i_{2} C_{3}-I I[n, m]\right) \mid r_{u}=3, r_{v}=1\right\}$, $R E_{2,2}=\left\{u v \in E\left(S i_{2} C_{3}-I I[n, m]\right) \mid r_{u}=2, r_{v}=2\right\}$, $R E_{2,1}=\left\{u v \in E\left(S i_{2} C_{3}-I I[n, m]\right) \mid r_{u}=2, r_{v}=1\right\}$, $R E_{1,1}=\left\{u v \in E\left(S i_{2} C_{3}-I I[n, m]\right) \mid r_{u}=1, r_{v}=1\right\}$, and cardinalities of partite sets are: $\left|R E_{3,2}\right|=2,\left|R E_{3,1}\right|=1$, $\left|R E_{2,2}\right|=2 n+2 m, \quad\left|R E_{2,1}\right|=8 n+8 m-14, \quad\left|R E_{1,1}\right|=$ $15 m n-13 n-13 m+11$

4. The $2 D$ structure of $G \cong S i_{2} C_{3}-I I I[n, m]$, $n, m \geq 1$, is shown in Figure 3 . Left picture is a unit cell and right is of $S i_{2} C_{3}-I I I[4,3]$ This structure consists of $n$ cells (in a row) and $m$ connected rows. Order of this graph $G$ is $10 \mathrm{~nm}$ and size is $15 \mathrm{~nm}-$ $2 n-3 m$.

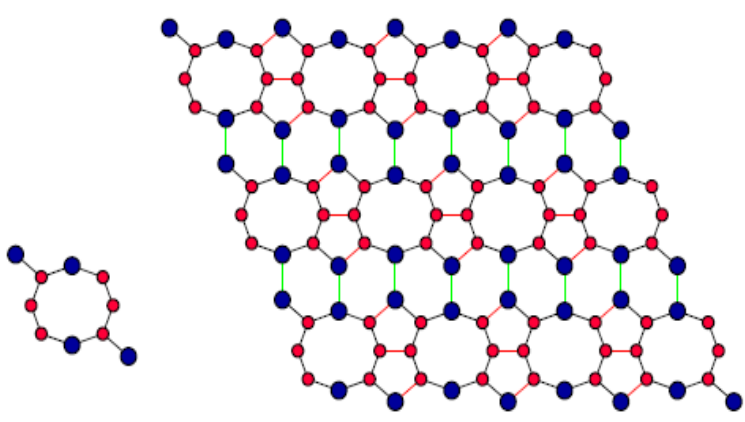

Figure 3

Edge set is partitioned into following sets:

$R E_{3,1}=\left\{u v \in E\left(S i_{2} C_{3}-I I I[n, m]\right) \mid r_{u}=3, r_{v}=1\right\}$, $R E_{2,2}=\left\{u v \in E\left(S i_{2} C_{3}-I I I[n, m]\right) \mid r_{u}=2, r_{v}=2\right\}$, $R E_{2,1}=\left\{u v \in E\left(S i_{2} C_{3}-I I I[n, m]\right) \mid r_{u}=2, r_{v}=1\right\}$, $R E_{1,1}=\left\{u v \in E\left(S i_{2} C_{3}-I I I[n, m]\right) \mid r_{u}=1, r_{v}=1\right\}$, And cardinalities of partite sets are $\left|R E_{3,1}\right|=1,\left|R E_{2,2}\right|=$ $2+2 m, \quad\left|R E_{2,1}\right|=8 n+8 m-12, \quad\left|R E_{1,1}\right|=15 m n-$ $10 n-13 m+8$

4. The $2 D$ structure of $G \cong S i C_{3}-I[n, m], n, m \geq 1$, is shown in Figure 4. This structure consists of $n$ cells (in a row) and $m$ connected rows.

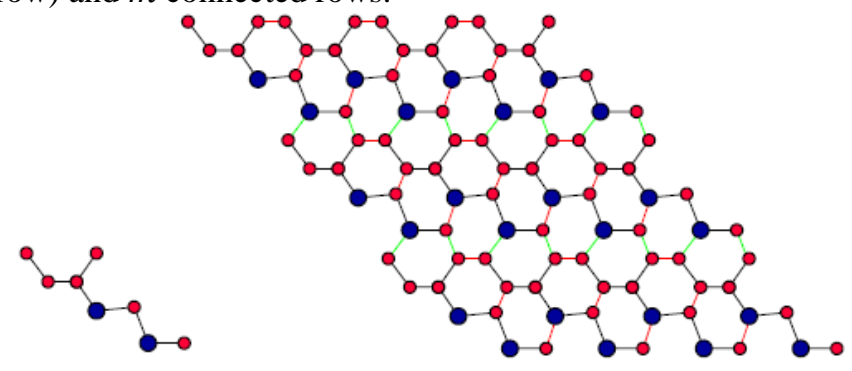

Figure 4

Left picture is a unit cell and right is of $\mathrm{SiC}_{3}-I[4,3]$. Order of this graph $G$ is $8 \mathrm{~nm}$ and size is $12 n m-2 n-$ $3 \mathrm{~m}$. Edge set is partitioned inteo following sets:

$$
R E_{3,2}=\left\{u v \in E\left(S_{i} C_{3}-I[n, m]\right) \mid r_{u}=3, r_{v}=2\right\}
$$

$R E_{3,1}=\left\{u v \in E\left(\operatorname{SiC}_{3}-I[n, m]\right) \mid r_{u}=3, r_{v}=1\right\},=1$

$R E_{2,2}=\left\{u v \in E\left(\mathrm{SiC}_{3}-I[n, m]\right) \mid r_{u}=2, r_{v}=2\right\}$,

$R E_{2,1}=\left\{u v \in E\left(\operatorname{SiC}_{3}-I[n, m]\right) \mid r_{u}=2, r_{v}=1\right\}$,

$R E_{1,1}=\left\{u v \in E\left(\mathrm{SiC}_{3}-I[n, m]\right) \mid r_{u}=1, r_{v}=1\right\}$,

And cardinalities are $\left|R E_{3,2}\right|=2,\left|R E_{3,1}\right|$

$$
\left|R E_{2,2}\right|=\left\{\begin{array}{c}
3 m-1 \text { for } n=1, m \geq 1 \\
2 n+2 m-3 \text { for } n>1, m \geq 1
\end{array}\right.
$$




$$
\begin{gathered}
\left|R E_{2,1}\right|=\left\{\begin{array}{c}
6 m-4 \text { for } n=1, m \geq 1 \\
4 n+8 m-8 \text { for } n>1, m \geq 1
\end{array}\right. \\
\left|R E_{1,1}\right|=\left\{\begin{array}{l}
12 m n-2 n-12 m+2 \text { for } n=1, m \geq 1 \\
12 m n-8 n-13 m+8 \text { for } n>1, m \geq 1
\end{array}\right.
\end{gathered}
$$

5. The $2 D$ structure of $\mathrm{SiC}_{3}-I I[n, m], n, m \geq 1$, is shown in Figure 5. This structure consists of $n$ cells (in a row) and $m$ connected rows. Order of this graph is $8 \mathrm{~nm}$ and size is $12 n m-2 n-2 m$. Left picture is a unit cell and right is of $\mathrm{SiC}_{3}-I I[4,3]$.

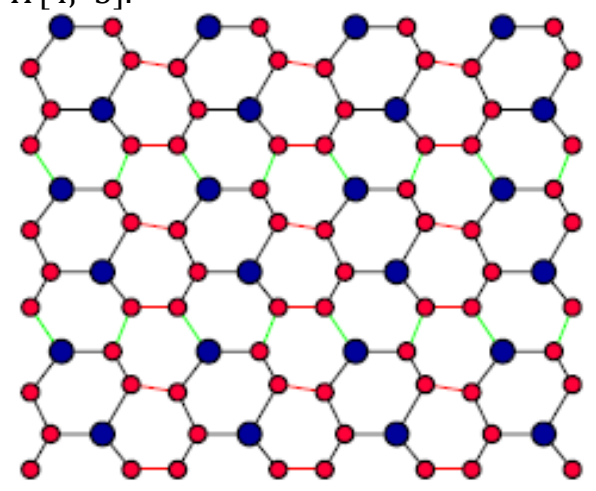

Figure 5

Edge set is partitioned into following sets

$R E_{3,1}=\left\{u v \in E\left(\operatorname{SiC}_{3}-I[n, m]\right) \mid r_{u}=3, r_{v}=1\right\}$,

$R E_{2,2}=\left\{u v \in E\left(\operatorname{SiC}_{3}-I[n, m]\right) \mid r_{u}=2, r_{v}=2\right\}$

$R E_{2,1}=\left\{u v \in E\left(S_{i C}-I[n, m]\right) \mid r_{u}=2, r_{v}=1\right\}$,

$R E_{1,1}=\left\{u v \in E\left(S_{i} C_{3}-I[n, m]\right) \mid r_{u}=1, r_{v}=1\right\}$,

And cardinalities of partite sets are computed as $\left|R E_{3,1}\right|=$ 2, $\left|R E_{2,2}\right|=2 n+1,\left|R E_{2,1}\right|=4 n+8 m-10,\left|R E_{1,1}\right|=$ $12 n m-8 n-10 m+7$.

6. The $2 D$ structure of $G \cong S i C_{3}-I I I[n, m], n, m \geq 1$, is shown in Figure 6.

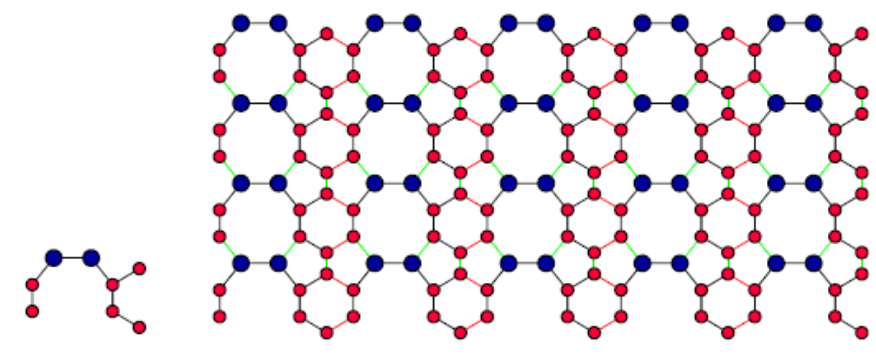

Figure 6

This structure consists of $n$ cells (in a row) and $m$ connected rows. Order of this graph is $8 \mathrm{~nm}$ and size is $12 \mathrm{~nm}-3 \mathrm{n}-$ $2 \mathrm{~m}$. Left picture is a unit cell and right is of $\mathrm{SiC}_{3}-$ III $[5,4]$.

Edge set is partitioned into following sets:

$R E_{3,2}=\left\{u v \in E\left(\mathrm{SiC}_{3}-I I I[n, m]\right) \mid r_{u}=3, r_{v}=2\right\}$,

$R E_{3,1}=\left\{u v \in E\left(\operatorname{SiC}_{3}-I I I[n, m]\right) \mid r_{u}=3, r_{v}=1\right\}$,

$R E_{2,2}=\left\{u v \in E\left(\operatorname{SiC}_{3}-I I I[n, m]\right) \mid r_{u}=2, r_{v}=2\right\}$,

$R E_{2,1}=\left\{u v \in E\left(\operatorname{SiC}_{3}-I I I[n, m]\right) \mid r_{u}=2, r_{v}=1\right\}$,

$R E_{1,1}=\left\{u v \in E\left(\operatorname{SiC}_{3}-I I I[n, m]\right) \mid r_{u}=1, r_{v}=1\right\}$,
And cardinalities of partite sets are $\left|R E_{3,2}\right|=2,\left|R E_{3,1}\right|=1$, $\left|R E_{2,2}\right|=3 n+2 m-3,\left|R E_{2,1}\right|=6 n+4 m-8,\left|R E_{1,1}\right|=$ $12 m n-12 n-8 m+8$.

7. The $2 D$ structure of $G \cong S i C_{4}-I[n, m], n, m \geq 1$, is shown in Figure 7. This structure consists of $n$ cells (in a row) and $m$ connected rows. Order of this graph $G$ is $10 \mathrm{~nm}$ and size is $15 n m-4 n-2 m+1$. Left picture is a unit cell and right is of $\mathrm{SiC}_{4}-I[4,3]$.

Partition of edge set is

$R E_{3,2}=\left\{u v \in E\left(\mathrm{SiC}_{4}-I[n, m]\right) \mid r_{u}=3, r_{v}=2\right\}$,

$R E_{3,1}=\left\{u v \in E\left(S_{i} C_{4}-I[n, m]\right) \mid r_{u}=3, r_{v}=1\right\}$,

$R E_{2,2}=\left\{u v \in E\left(S i C_{4}-I[n, m]\right) \mid r_{u}=2, r_{v}=2\right\}$,

$R E_{2,1}=\left\{u v \in E\left(\mathrm{SiC}_{4}-I[n, m]\right) \mid r_{u}=2, r_{v}=1\right\}$,

$R E_{1,1}=\left\{u v \in E\left(\mathrm{SiC}_{4}-I[n, m]\right) \mid r_{u}=1, r_{v}=1\right\}$,

And cardinalities of partite sets are $\left|R E_{3,2}\right|=2,\left|R E_{3,1}\right|=$ $3 n-2,\left|R E_{2,2}\right|=n+2 m-2, \quad\left|R E_{2,1}\right|=2 n+4 m-2$, $\left|R E_{1,1}\right|=14 m n-10 n-8 m+5$

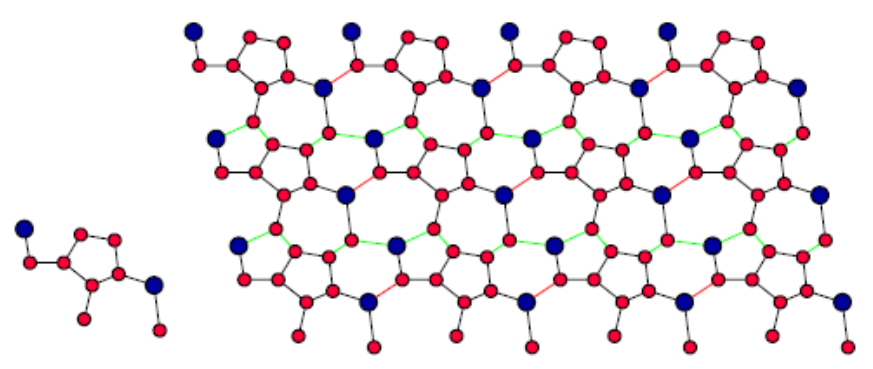

Figure 7

8. The $2 D$ structure of $\mathrm{SiC}_{4}-I I[n, m], n, m \geq 1$, is shown in Figure 8.

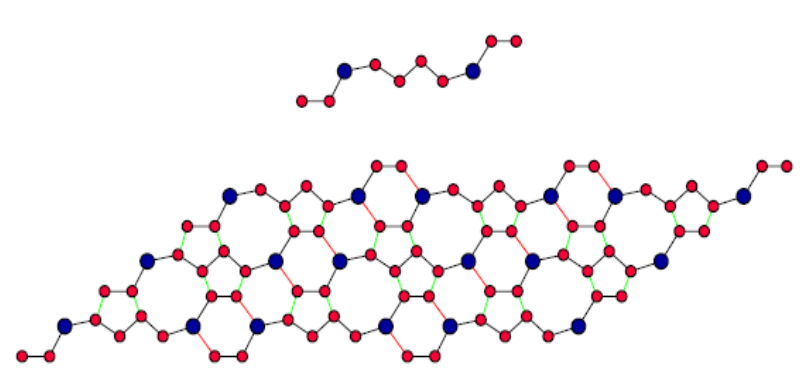

Figure 8

This structure consists of $n$ cells (in a row) and $m$ connected rows. Order of this graph is $10 \mathrm{~nm}$ and size is $15 \mathrm{~nm}-$ $4 n-2 m$. First picture is a unit cell and second is of $\mathrm{SiC}_{4}-$ $I I[3,3]$. Edge set is partitioned into following sets: $R E_{3,2}=\left\{u v \in E\left(\operatorname{SiC}_{4}-I I[n, m]\right) \mid r_{u}=3, r_{v}=2\right\}$, $R E_{2,2}=\left\{u v \in E\left(S_{i C}-I I[n, m]\right) \mid r_{u}=2, r_{v}=2\right\}$, $R E_{2,1}=\left\{u v \in E\left(\mathrm{SiC}_{4}-I I[n, m]\right) \mid r_{u}=2, r_{v}=1\right\}$, $R E_{1,1}=\left\{u v \in E\left(\operatorname{SiC}_{4}-I I[n, m]\right) \mid r_{u}=1, r_{v}=1\right\}$, And cardinalities of partite sets are $\left|R E_{3,2}\right|=2$

$$
\left|R E_{2,2}\right|=\left\{\begin{array}{l}
5 n+2 \text { for } m=1, n \geq 1 \\
2 n+2 \text { for } m>1, n \geq 1
\end{array}\right.
$$




$$
\begin{gathered}
\left|R E_{2,1}\right|= \begin{cases}6 n-6 \quad \text { for } m=1, n \geq 1 \\
12 n+8 m-14 \text { for } m>1, n \geq 1\end{cases} \\
\left|R E_{1,1}\right|=\left\{\begin{array}{c}
15 n m-15 n-2 m+2 \text { for } m=1, n \geq 1 \\
15 n m-18 n-10 m+10 \text { for } m>1, n \geq 1
\end{array}\right.
\end{gathered}
$$

\section{MAIN RESULTS}

In this section, we prove results about family of silicon carbide graphs listed in previous section. Here we present results of Revan indices and Revan polynomials for our list of silicon carbide graphs.

Theorem 3.1. Let $G \cong S i_{2} C_{3}-I[n, m], n, m \geq 1$ be the silicon carbide graph, then

$$
\begin{array}{ll}
\text { 1. } & R_{1}(G)=30 m n+4 n+6 m-4 . \\
\text { 2. } & R_{2}(G)=15 m n+7 n+11 m-2 . \\
\text { 3. } & R_{3}(G)=6 n+8 m-6 .
\end{array}
$$

Proof. Adding entries of last column, we get $R_{1}(G)$.

\begin{tabular}{|c|c|c|c|}
\hline$\left\{r_{u}, r_{v}\right\}$ & $f=\left|R E_{u, v}\right|$ & $\begin{array}{c}r_{u} \\
+r_{v}\end{array}$ & $f \cdot\left(r_{u}+r_{v}\right)$ \\
\hline$\{3,2\}$ & 1 & 5 & 5 \\
\hline$\{3,1\}$ & 1 & 4 & 4 \\
\hline$\{2,2\}$ & $n+2 m$ & 4 & $4 n+8 m$ \\
\hline$\{2,1\}$ & $6 n+8 m-9$ & 3 & $18 n+24 m-27$ \\
\hline$\{1,1\}$ & $15 m n-9 n$ & 2 & $30 m n-18 n$ \\
& $-13 m+7$ & & $-26 m+14$ \\
\hline
\end{tabular}

Revan first polynomial is

$$
\begin{aligned}
& R_{1}(G, x)=\sum_{u v \in E(G)} x^{\left(r_{u}+r_{v}\right)}=x^{5}+(n+2 m+1) x^{4} \\
& +(6 n+8 m-9) x^{3}+(15 m n-9 n-13 m+7) x^{2} .
\end{aligned}
$$

Adding entries of last column, we get $R_{2}(G)$

\begin{tabular}{|c|c|c|c|}
\hline$\left\{r_{u}, r_{v}\right\}$ & $f=\left|R E_{u, v}\right|$ & $r_{u} \cdot r_{v}$ & $f \cdot\left(r_{u} \cdot r_{v}\right)$ \\
\hline$\{3,2\}$ & 1 & 6 & 6 \\
\hline$\{3,1\}$ & 1 & 3 & 3 \\
\hline$\{2,2\}$ & $n+2 m$ & 4 & $4 n+8 m$ \\
\hline$\{2,1\}$ & $6 n+8 m-9$ & 2 & $12 n+16 m-18$ \\
\hline$\{1,1\}$ & $15 m n-9 n$ & 1 & $15 m n-9 n$ \\
& $-13 m+7$ & & $-13 m+7$ \\
\hline
\end{tabular}

Revan second polynomial is

$R_{2}(G, x)=\sum_{u v \in E(G)} x^{\left(r_{u} \cdot r_{v}\right)}=x^{6}+(n+2 m) x^{4}+x^{3}+$ $(6 n+8 m-9) x^{2}+(15 m n-9 n-13 m+7) x$

Adding entries of last column, we get $R_{3}(G)$

\begin{tabular}{|c|c|c|c|}
\hline$\left\{r_{u}, r_{v}\right\}$ & $f=\left|R E_{u, v}\right|$ & $\begin{array}{c}\mid r_{u} \\
-r_{v} \mid\end{array}$ & $f \cdot\left|r_{u}-r_{v}\right|$ \\
\hline$\{3,2\}$ & 1 & 1 & 1 \\
\hline$\{3,1\}$ & 1 & 2 & 2 \\
\hline$\{2,2\}$ & $n+2 m$ & 0 & 0 \\
\hline$\{2,1\}$ & $6 n+8 m-9$ & 1 & $6 n+8 m-9$ \\
\hline$\{1,1\}$ & $\begin{array}{c}15 m n-9 n \\
-13 m+7\end{array}$ & 0 & 0 \\
\hline
\end{tabular}

Revan third polynomial is

$R_{3}(G, x)=\sum_{u v \in E(G)} x^{\left|r_{u}-r_{v}\right|}=x^{2}+(6 n+8 m-8) x+$ $(15 m n-8 n-11 m+7)$

Theorem 3.2. Let $G \cong S i_{2} C_{3}-I I[n, m], n, m \geq 1$ be the silicon carbide graph, then

$$
\begin{array}{ll}
\text { 1. } & R_{1}(G)=30 m n+6 n+6 m-6 . \\
\text { 2. } & R_{2}(G)=15 m n+11 n+11 m-2 . \\
\text { 3. } & R_{3}(G)=8 n+8 m-10 .
\end{array}
$$

Proof. Adding entries of last column, we get $R_{1}(G)$

\begin{tabular}{|c|c|c|c|}
\hline$\left\{r_{u}, r_{v}\right\}$ & $f=\left|R E_{u, v}\right|$ & $\begin{array}{c}r_{u} \\
+r_{v}\end{array}$ & $f \cdot\left(r_{u}+r_{v}\right)$ \\
\hline$\{3,2\}$ & 2 & 5 & 10 \\
\hline$\{3,1\}$ & 1 & 4 & 4 \\
\hline$\{2,2\}$ & $2 n+2 m$ & 4 & $8 n+8 m$ \\
\hline$\{2,1\}$ & $8 n+8 m-14$ & 3 & $24 n+24 m-42$ \\
\hline$\{1,1\}$ & $\begin{array}{c}15 m n-13 n \\
-13 m+11\end{array}$ & 2 & $\begin{array}{c}30 m n-26 n \\
-26 m+22\end{array}$ \\
\hline
\end{tabular}

Revan first polynomial is

$$
\begin{aligned}
& R_{1}(G, x)=\sum_{u v \in E(G)} x^{\left(r_{u}+r_{v}\right)}=2 x^{5}+(2 n+2 m+1) x^{4} \\
& +(8 n+8 m-14) x^{3}+(15 m n-13 n-13 m+11) x^{2} .
\end{aligned}
$$

Adding entries of last column, we get $R_{2}(G)$

\begin{tabular}{|c|c|c|c|}
\hline$\left\{r_{u}, r_{v}\right\}$ & $f=\left|R E_{u, v}\right|$ & $r_{u} \cdot r_{v}$ & $f \cdot\left(r_{u} \cdot r_{v}\right)$ \\
\hline$\{3,2\}$ & 2 & 6 & 12 \\
\hline$\{3,1\}$ & 1 & 3 & 3 \\
\hline$\{2,2\}$ & $2 n+2 m$ & 4 & $8 n+8 m$ \\
\hline$\{2,1\}$ & $8 n+8 m-14$ & 2 & $16 n+16 m-28$ \\
\hline$\{1,1\}$ & $15 m n-13 n$ & 1 & $15 m n-13 n$ \\
& $-13 m+11$ & & $-13 m+11$ \\
\hline
\end{tabular}

Revan second polynomial is $R_{2}(G, x)=\sum_{u v \in E(G)} x^{\left(r_{u} \cdot r_{v}\right)}=$ $2 x^{6}+(2 n+2 m) x^{4}+x^{3}+$

$$
(8 n+8 m-14) x^{2}+(15 m n-13 n-13 m+11) x
$$

Adding entries of last column, we get $R_{3}(G)$

\begin{tabular}{|c|c|c|c|}
\hline$\left\{r_{u}, r_{v}\right\}$ & $f=\left|R E_{u, v}\right|$ & $\begin{array}{c}\mid r_{u} \\
-r_{v} \mid\end{array}$ & $f \cdot\left|r_{u}-r_{v}\right|$ \\
\hline$\{3,2\}$ & 2 & 1 & 2 \\
\hline$\{3,1\}$ & 1 & 2 & 2 \\
\hline$\{2,2\}$ & $2 n+2 m$ & 0 & 0 \\
\hline$\{2,1\}$ & $\begin{array}{l}8 n+8 m \\
-14\end{array}$ & 1 & $8 n+8 m-14$ \\
\hline$\{1,1\}$ & $\begin{array}{c}15 m n-13 n \\
-13 m+11\end{array}$ & 0 & 0 \\
\hline
\end{tabular}

Revan third polynomial is $R_{3}(G, x)=\sum_{u v \in E(G)} x^{\left|r_{u}-r_{v}\right|}=$ $x^{2}+(8 n+8 m-12) x+(15 m n-11 n-11 m+11)$.

Theorem 3.3. Let $G \cong S i_{2} C_{3}-I I I[n, m], n, m \geq 1$ be the silicon carbide graph, then
1.
2. $R_{2}(G)=15 m n+6 n+11 m-2$.
3. $\quad R_{3}(G)=8 n+8 m-8$. 
Proof. Adding entries of last column, we get $R_{1}(G)$.

\begin{tabular}{|c|c|c|c|}
\hline$\left\{r_{u}, r_{v}\right\}$ & $f=\left|R E_{u, v}\right|$ & $\begin{array}{c}r_{u} \\
+r_{v}\end{array}$ & $f \cdot\left(r_{u}+r_{v}\right)$ \\
\hline$\{3,1\}$ & 2 & 4 & 8 \\
\hline$\{2,2\}$ & $2 m+2$ & 4 & $8 m+8$ \\
\hline$\{2,1\}$ & $8 n+8 m-12$ & 3 & $24 n+24 m-36$ \\
\hline$\{1,1\}$ & $\begin{array}{c}15 m n-10 n \\
-13 m+8\end{array}$ & 2 & $\begin{array}{l}30 m n-20 n \\
-26 m+16\end{array}$ \\
\hline
\end{tabular}

Revan first polynomial is

$R_{1}(G, x)=\sum_{u v \in E(G)} x^{\left(r_{u}+r_{v}\right)}=(2 m+4) x^{4}+(8 n+8 m-$ 12) $x^{3}+(15 m n-10 n-13 m+8) x^{2}$.

Adding entries of last column, we get $R_{2}(G)$

\begin{tabular}{|c|c|c|c|}
\hline$\left\{r_{u}, r_{v}\right\}$ & $f=\left|R E_{u, v}\right|$ & $r_{u} \cdot r_{v}$ & $f \cdot\left(r_{u} \cdot r_{v}\right)$ \\
\hline$\{3,1\}$ & 2 & 3 & 6 \\
\hline$\{2,2\}$ & $2 m+2$ & 4 & $8 m+8$ \\
\hline$\{2,1\}$ & $8 n+8 m-12$ & 2 & $16 n+16 m-24$ \\
\hline$\{1,1\}$ & $\begin{array}{c}15 m n-10 n \\
-13 m+8\end{array}$ & 1 & $\begin{array}{c}15 m n-10 n \\
-13 m+8\end{array}$ \\
\hline
\end{tabular}

Revan second polynomial is

$$
\begin{aligned}
& R_{2}(G, x)=\sum_{u v \in E(G)} x^{\left(r_{u} \cdot r_{v}\right)}=(2 m+4) x^{4}+2 x^{3}+ \\
& (8 n+8 m-12) x^{2}+(15 m n-10 n-13 m+8) x
\end{aligned}
$$

Adding entries of last column, we get $R_{3}(G)$

\begin{tabular}{|c|c|c|c|}
\hline$\left\{r_{u}, r_{v}\right\}$ & $f=\left|R E_{u, v}\right|$ & $\begin{array}{c}\mid r_{u} \\
-r_{v} \mid\end{array}$ & $f \cdot\left|r_{u}-r_{v}\right|$ \\
\hline$\{3,1\}$ & 2 & 2 & 4 \\
\hline$\{2,2\}$ & $2 m+2$ & 0 & 0 \\
\hline$\{2,1\}$ & $\begin{array}{l}8 n+8 m \\
-12\end{array}$ & 1 & $8 n+8 m-12$ \\
\hline$\{1,1\}$ & $\begin{array}{l}15 m n-10 n \\
-13 m+8\end{array}$ & 0 & 0 \\
\hline
\end{tabular}

Revan third polynomial is $R_{3}(G, x)=\sum_{u v \in E(G)} x^{\left|r_{u}-r_{v}\right|}=$ $2 x^{2}+(8 n+8 m-12) x+(15 m n-10 n-11 m+10)$.

Theorem 3.4. Let $\mathrm{SiC}_{3}-I[n, m], n, m \geq 1$ be the silicon carbide graph, then

1. $R_{1}(G)=$

$\{24 m n-4 n+6 m+2$ for $n=1, m \geq 1$

$24 m n+4 n+6 m-8$ for $n>1, m \geq 1$

2. $R_{2}(G)=$

$\{12 m n-2 n+12 m+5$ for $n=1, m \geq 1$

$\{12 m n+8 n+11 m-8$ for $n>1, m \geq 1$

3. $R_{3}(G)= \begin{cases}6 m & \text { for } n=1, m \geq 1 \\ 4 n+8 m-4 & \text { for } n>1, m \geq 1\end{cases}$

Proof. Adding entries of last column, we get $R_{1}(G)$ for for $n=1, m \geq 1$.

\begin{tabular}{|c|c|c|c|}
\hline$\left\{r_{u}, r_{v}\right\}$ & $f=\left|R E_{u, v}\right|$ & $\begin{array}{c}r_{u} \\
+r_{v}\end{array}$ & $f \cdot\left(r_{u}+r_{v}\right)$ \\
\hline$\{3,2\}$ & 2 & 5 & 10 \\
\hline$\{3,1\}$ & 1 & 4 & 4 \\
\hline$\{2,2\}$ & $3 m-1$ & 4 & $12 m-4$ \\
\hline$\{2,1\}$ & $6 m-4$ & 3 & $18 m-12$ \\
\hline$\{1,1\}$ & $\begin{array}{c}12 m n-2 n \\
-12 m+2\end{array}$ & 2 & $\begin{array}{l}24 m n-4 n \\
-24 m+4\end{array}$ \\
\hline
\end{tabular}

Adding entries of last column, we get $R_{1}(G)$ for $n>1$, $m \geq 1$.

\begin{tabular}{|l|c|c|c|}
\hline$\left\{r_{u}, r_{v}\right\}$ & $f=\left|R E_{u, v}\right|$ & $\begin{array}{c}r_{u} \\
+r_{v}\end{array}$ & $f \cdot\left(r_{u}+r_{v}\right)$ \\
\hline$\{3,2\}$ & 2 & 5 & 10 \\
\hline$\{3,1\}$ & 1 & 4 & 4 \\
\hline$\{2,2\}$ & $2 n+2 m-3$ & 4 & $8 n+8 m-12$ \\
\hline$\{2,1\}$ & $4 n+8 m-8$ & 3 & $12 n+24 m-24$ \\
\hline$\{1,1\}$ & $12 m n-8 n$ & 2 & $24 m n-16 n$ \\
& $-13 m+8$ & & $-26 m+16$ \\
\hline
\end{tabular}

Revan first polynomial is $R_{1}(G, x)$ for $n=1, m \geq 1$

$\sum_{u v \in E(G)} x^{\left(r_{u}+r_{v}\right)}=2 x^{5}+3 m x^{4}+(6 m-4) x^{3}+(12 m n-$ $2 n-12 m+2) x^{2}$.

And Revan first polynomial for $n>1, m \geq 1$

$R_{1}(G, x)=2 x^{5}+(2 n+2 m-2) x^{4}+(4 n+8 m-8) x^{3}+$ $(12 m n-8 n-13 m+8) x^{2}$.

Adding entries of last column, we get $R_{2}(G)$ for $n=1, m \geq 1$

\begin{tabular}{|c|c|c|c|}
\hline$\left\{r_{u}, r_{v}\right\}$ & $f=\left|R E_{u, v}\right|$ & $r_{u} \cdot r_{v}$ & $f \cdot\left(r_{u} \cdot r_{v}\right)$ \\
\hline$\{3,2\}$ & 2 & 6 & 12 \\
\hline$\{3,1\}$ & 1 & 3 & 3 \\
\hline$\{2,2\}$ & $3 m-1$ & 4 & $12 m-4$ \\
\hline$\{2,1\}$ & $6 m-4$ & 2 & $12 m-8$ \\
\hline$\{1,1\}$ & $12 m n-2 n$ & 1 & $12 m n-2 n$ \\
& $-12 m+2$ & & $-12 m+2$ \\
\hline
\end{tabular}

Adding entries of last column, we get $R_{2}(G)$ for $n>1$, $m \geq 1$.

\begin{tabular}{|c|c|c|c|}
\hline$\left\{r_{u}, r_{v}\right\}$ & $f=\left|R E_{u, v}\right|$ & $r_{u} \cdot r_{v}$ & $f \cdot\left(r_{u} \cdot r_{v}\right)$ \\
\hline$\{3,2\}$ & 2 & 6 & 12 \\
\hline$\{3,1\}$ & 1 & 3 & 3 \\
\hline$\{2,2\}$ & $2 n+2 m-3$ & 4 & $8 n+8 m-12$ \\
\hline$\{2,1\}$ & $4 n+8 m-8$ & 2 & $8 n+8 m-16$ \\
\hline$\{1,1\}$ & $12 m n-8 n$ & 1 & $12 m n-8 n$ \\
& $-13 m+8$ & & $-13 m+8$ \\
\hline
\end{tabular}

Revan second polynomial $R_{2}(G, x)$ for $n=1, m \geq 1$

$\sum_{u v \in E(G)} x^{\left(r_{u} \cdot r_{v}\right)}=2 x^{6}+(3 m-1) x^{4}+x^{3}+$ $+(6 m-4) x^{2}+(12 m n-2 n-12 m+2) x$.

And Revan second polynomial for $n>1, m \geq 1$ is

$R_{2}(G, x)=2 x^{6}+(2 n+2 m-3) x^{4}+x^{3}+$

$+(4 n+8 m-8) x^{2}+(12 m n-8 n-13 m+8) x$.

Adding entries of last column, we get

$R_{3}(G)$ for $n=1, m \geq 1$. 


\begin{tabular}{|c|c|c|c|}
\hline$\left\{r_{u}, r_{v}\right\}$ & $f=\left|R E_{u, v}\right|$ & $\begin{array}{c}\mid r_{u} \\
-r_{v} \mid\end{array}$ & $f \cdot\left|r_{u}-r_{v}\right|$ \\
\hline$\{3,2\}$ & 2 & 1 & 2 \\
\hline$\{3,1\}$ & 1 & 2 & 2 \\
\hline$\{2,2\}$ & $3 m-1$ & 0 & 0 \\
\hline$\{2,1\}$ & $6 m-4$ & 1 & $6 m-4$ \\
\hline$\{1,1\}$ & $\begin{array}{l}12 m n-2 n \\
-12 m+2\end{array}$ & 0 & 0 \\
\hline
\end{tabular}

Adding entries of last column, we get $R_{3}(G)$ for $n>1$, $m \geq 1$.

\begin{tabular}{|c|c|c|c|}
\hline$\left\{r_{u}, r_{v}\right\}$ & $f=\left|R E_{u, v}\right|$ & $\begin{array}{c}\mid r_{u} \\
-r_{v} \mid\end{array}$ & $f \cdot\left|r_{u}-r_{v}\right|$ \\
\hline$\{3,2\}$ & 2 & 1 & 2 \\
\hline$\{3,1\}$ & 1 & 2 & 2 \\
\hline$\{2,2\}$ & $2 n+2 m-3$ & 0 & 0 \\
\hline$\{2,1\}$ & $4 n+8 m-8$ & 1 & $4 n+8 m-8$ \\
\hline$\{1,1\}$ & $\begin{array}{c}12 m n-8 n \\
-13 m+8\end{array}$ & 0 & 0 \\
\hline
\end{tabular}

Revan second polynomial is $R_{2}(G, x)=\sum_{u v \in E(G)} x^{\left(r_{u} \cdot r_{v}\right)}=$ $(2 n+1) x^{4}+x^{3}+(4 n+8 m-10) x^{2}+(12 m n-8 n-$ $10 m+7) x$

Adding entries of last column, we get $R_{3}(G)$

\begin{tabular}{|c|c|c|c|}
\hline$\left\{r_{u}, r_{v}\right\}$ & $f=\left|R E_{u, v}\right|$ & $\begin{array}{c}\mid r_{u} \\
-r_{v} \mid\end{array}$ & $f \cdot\left|r_{u}-r_{v}\right|$ \\
\hline$\{3,1\}$ & 2 & 2 & 4 \\
\hline$\{2,2\}$ & $2 n+1$ & 0 & 0 \\
\hline$\{2,1\}$ & $\begin{array}{l}4 n+8 m \\
-10\end{array}$ & 1 & $4 n+8 m-10$ \\
\hline$\{1,1\}$ & $\begin{array}{c}12 m n-8 n \\
-10 m+7\end{array}$ & 0 & 0 \\
\hline
\end{tabular}

Revan third polynomial is $R_{3}(G, x)=\sum_{u v \in E(G)} x^{\left|r_{u}-r_{v}\right|}=$ $2 x^{2}+4 n+8 m-10 x+(12 m n-6 n-10 m+8)$.

Theorem 3.6. Let $\mathrm{SiC}_{3}-I I I[n, m], n, m \geq 1$ be the silicon carbide graph, then

$$
\begin{array}{ll}
\text { 1. } & R_{1}(G)=24 m n+6 n+4 m-6 . \\
\text { 2. } & R_{2}(G)=12 m n+12 n+8 m-5 . \\
\text { 3. } & R_{3}(G)=6 n+4 m-4 .
\end{array}
$$

$=\sum_{u v \in E(G)} x^{\left|r_{u}-r_{v}\right|}=x^{2}+(6 m-2) x+(12 m n-2 n-$ $9 m+1)$.

And Revan third polynomial for $n>1, m \geq 1$

$R_{3}(G, x)=x^{2}+(4 n+8 m-6) x+(12 m n-6 n-$ $11 m+5)$.

Theorem 3.5. Let $\mathrm{SiC}_{3}-I I[n, m], n, m \geq 1$ be the silicon carbide graph, then
1. $R_{1}(G)=24 m n+4 n+4 m-8$.
2. $R_{2}(G)=12 m n+8 n+6 m-6$.
3. $R_{3}(G)=4 n+8 m-6$.

Proof. Adding entries of last column, we get $R_{1}(G)$.

\begin{tabular}{|c|c|c|c|}
\hline$\left\{r_{u}, r_{v}\right\}$ & $f=\left|R E_{u, v}\right|$ & $\begin{array}{c}r_{u} \\
+r_{v}\end{array}$ & $f \cdot\left(r_{u}+r_{v}\right)$ \\
\hline$\{3,1\}$ & 1 & 4 & 4 \\
\hline$\{2,2\}$ & $2 n+1$ & 4 & $8 n+4$ \\
\hline$\{2,1\}$ & $4 n+8 m-10$ & 3 & $12 n+24 m-30$ \\
\hline$\{1,1\}$ & $12 m n-8 n$ & 2 & $24 m n-8 n$ \\
& $-10 m+7$ & & $-10 m+7$ \\
\hline
\end{tabular}

Revan first polynomial is $R_{1}(G, x)=\sum_{u v \in E(G)} x^{\left(r_{u}+r_{v}\right)}=$ $(2 n+1) x^{4}+(4 n+8 m-10) x^{3}+(12 m n-8 n-10 m+$ 7) $x^{2}$.

Adding entries of last column, we get $R_{2}(G)$

\begin{tabular}{|c|c|c|c|}
\hline$\left\{r_{u}, r_{v}\right\}$ & $f=\left|R E_{u, v}\right|$ & $r_{u} \cdot r_{v}$ & $f \cdot\left(r_{u} \cdot r_{v}\right)$ \\
\hline$\{3,1\}$ & 1 & 3 & 3 \\
\hline$\{2,2\}$ & $2 n+1$ & 4 & $8 n+4$ \\
\hline$\{2,1\}$ & $4 n+8 m-10$ & 2 & $8 n+16 m-20$ \\
\hline$\{1,1\}$ & $12 m n-8 n$ & 1 & $12 m n-8 n$ \\
& $-10 m+7$ & & $-10 m+7$ \\
\hline
\end{tabular}

Proof. Adding entries of last column, we get $R_{1}(G)$.

\begin{tabular}{|l|l|l|c|}
\hline$\left\{r_{u}, r_{v}\right\}$ & $f=\left|R E_{u, v}\right|$ & $\begin{array}{l}r_{u} \\
+r_{v}\end{array}$ & $f \cdot\left(r_{u}+r_{v}\right)$ \\
\hline$\{3,2\}$ & 2 & 5 & 10 \\
\hline$\{3,1\}$ & 1 & 4 & 4 \\
\hline$\{2,2\}$ & $3 n+2 m-3$ & 4 & $12 n+8 m-12$ \\
\hline$\{2,1\}$ & $6 n+4 m-8$ & 3 & $18 n+12 m-24$ \\
\hline$\{1,1\}$ & $12 m n-12 n$ & 2 & $24 m n-24 n$ \\
& $-8 m+8$ & & $-16 m+16$ \\
\hline
\end{tabular}

Revan first polynomial is $R_{1}(G, x)=\sum_{u v \in E(G)} x^{\left(r_{u}+r_{v}\right)}=$ $2 x^{5}+(3 n+2 m-3) x^{4}$

$+(6 n+4 m-8) x^{3}+(12 m n-12 n-8 m+8) x^{2}$.

Adding entries of last column, we get $R_{2}(G)$

\begin{tabular}{|c|c|c|c|}
\hline$\left\{r_{u}, r_{v}\right\}$ & $f=\left|R E_{u, v}\right|$ & $r_{u} \cdot r_{v}$ & $f \cdot\left(r_{u} \cdot r_{v}\right)$ \\
\hline$\{3,2\}$ & 2 & 6 & 12 \\
\hline$\{3,1\}$ & 1 & 3 & 3 \\
\hline$\{2,2\}$ & $3 n+2 m-3$ & 4 & $12 n+8 m-12$ \\
\hline$\{2,1\}$ & $6 n+4 m-8$ & 2 & $12 n+8 m-16$ \\
\hline$\{1,1\}$ & $12 m n-12 n$ & 1 & $12 m n-12 n$ \\
& $-8 m+8$ & & $-8 m+8$ \\
\hline
\end{tabular}

Revan second polynomial is $R_{2}(G, x)=\sum_{u v \in E(G)} x^{\left(r_{u} \cdot r_{v}\right)}=$ $2 x^{6}+(3 n+2 m-3) x^{4}+x^{3}++(6 n+4 m-8) x^{2}+$ $(12 m n-12 n-8 m+8) x$ 
Adding entries of last column, we get $R_{3}(G)$

\begin{tabular}{|c|c|c|c|}
\hline$\left\{r_{u}, r_{v}\right\}$ & $f=\left|R E_{u, v}\right|$ & $\begin{array}{c}\mid r_{u} \\
-r_{v} \mid\end{array}$ & $f \cdot\left|r_{u}-r_{v}\right|$ \\
\hline$\{3,2\}$ & 2 & 1 & 2 \\
\hline$\{3,1\}$ & 1 & 2 & 2 \\
\hline$\{2,2\}$ & $3 n+2 m-3$ & 0 & 0 \\
\hline$\{2,1\}$ & $6 n+4 m-8$ & 1 & $6 n+4 m-8$ \\
\hline$\{1,1\}$ & $\begin{array}{l}12 m n-12 n \\
-8 m+8\end{array}$ & 0 & 0 \\
\hline
\end{tabular}

Revan third polynomial is $R_{3}(G, x)=\sum_{u v \in E(G)} x^{\left|r_{u}-r_{v}\right|}=$ $x^{2}+(6 n+4 m-6) x+(12 m n-9 n-6 m+5)$.

Theorem 3.7. Let $\mathrm{SiC}_{4}-I[n, m], n, m \geq 1$ be the silicon carbide graph, then
1. $R_{1}(G)=30 m n+2 n+4 m-2$.
2. $R_{2}(G)=15 m n+7 n+8 m-1$.
3. $R_{3}(G)=8 n+4 m-4$.

Proof. Adding entries of last column, we get $R_{1}(G)$.

\begin{tabular}{|c|c|c|c|}
\hline$\left\{r_{u}, r_{v}\right\}$ & $f=\left|R E_{u, v}\right|$ & $\begin{array}{c}r_{u} \\
+r_{v}\end{array}$ & $f \cdot\left(r_{u}+r_{v}\right)$ \\
\hline$\{3,2\}$ & 2 & 5 & 10 \\
\hline$\{3,1\}$ & $3 n-2$ & 4 & $12 n-8$ \\
\hline$\{2,2\}$ & $n+2 m-2$ & 4 & $4 n+8 m-8$ \\
\hline$\{2,1\}$ & $2 n+4 m-2$ & 3 & $6 n+12 m-6$ \\
\hline$\{1,1\}$ & $\begin{array}{l}15 m n-10 n \\
-8 m+5\end{array}$ & 2 & $\begin{array}{l}30 m n-20 n \\
-16 m+10\end{array}$ \\
\hline
\end{tabular}

Revan first polynomial is $R_{1}(G, x)=\sum_{u v \in E(G)} x^{\left(r_{u}+r_{v}\right)}=$ $2 x^{5}+(n+2 m-2) x^{4}$

$+(2 n+4 m-2) x^{3}+(15 m n-10 n-8 m+5) x^{2}$.

Adding entries of last column, we get $R_{2}(G)$

\begin{tabular}{|c|c|c|c|}
\hline$\left\{r_{u}, r_{v}\right\}$ & $f=\left|R E_{u, v}\right|$ & $r_{u} \cdot r_{v}$ & $f \cdot\left(r_{u} \cdot r_{v}\right)$ \\
\hline$\{3,2\}$ & 2 & 6 & 12 \\
\hline$\{3,1\}$ & $3 n-2$ & 3 & $9 n-6$ \\
\hline$\{2,2\}$ & $n+2 m-2$ & 4 & $4 n+8 m-8$ \\
\hline$\{2,1\}$ & $2 n+4 m-2$ & 2 & $4 n+8 m-4$ \\
\hline$\{1,1\}$ & $\begin{array}{l}15 m n-10 n \\
-8 m+5\end{array}$ & 1 & $\begin{array}{l}15 m n-10 n \\
-8 m+5\end{array}$ \\
\hline
\end{tabular}

Revan second polynomial is

$R_{2}(G, x)=\sum_{u v \in E(G)} x^{\left(r_{u} \cdot r_{v}\right)}=2 x^{6}+(n+2 m-2) x^{4}+$ $(3 n-2) x^{3}++(2 n+4 m-2) x^{2}+(15 m n-10 n-$ $8 m+5) x$

Adding entries of last column, we get $R_{3}(G)$

\begin{tabular}{|c|c|c|c|}
\hline$\left\{r_{u}, r_{v}\right\}$ & $f=\left|R E_{u, v}\right|$ & $\begin{array}{c}\mid r_{u} \\
-r_{v} \mid\end{array}$ & $f \cdot\left|r_{u}-r_{v}\right|$ \\
\hline$\{3,2\}$ & 2 & 1 & 2 \\
\hline$\{3,1\}$ & $3 n-2$ & 2 & $6 n-4$ \\
\hline$\{2,2\}$ & $n+2 m-2$ & 0 & 0 \\
\hline$\{2,1\}$ & $2 n+4 m-2$ & 1 & $2 n+4 m-2$ \\
\hline$\{1,1\}$ & $\begin{array}{l}15 m n-10 n \\
-8 m+5\end{array}$ & 0 & 0 \\
\hline
\end{tabular}

Revan third polynomial is $R_{3}(G, x)=\sum_{u v \in E(G)} x^{\left|r_{u}-r_{v}\right|}=$ $(3 n-2) x^{2}+(2 n+4 m) x+(15 m n-9 n-6 m+3)$.

Theorem 3.8. Let $\mathrm{Si}_{4}-I I[n, m], n, m \geq 1$ be the silicon carbide graph, then

1. $R_{1}(G)=$

$\{30 m n+8 n-4 m+4$ for $m=1, n \geq 1$

$\{30 m n+8 n+4 m-4$ for $m>1, n \geq 1$

$$
\text { 2. } R_{2}(G)=
$$

$\{15 m n+17 n-2 m+10$ for $m=1, n \geq 1$

$\{15 m n-14 n+6 m+2$ for $m>1, n \geq 1$

$$
\text { 3. } R_{3}(G)= \begin{cases}6 n-4 & \text { for } m=1, n \geq 1 \\ 12 n+8 m-12 & \text { for } m>1, n \geq 1\end{cases}
$$

Proof.

Proof. Adding entries of last column, we get $R_{1}(G)$ for $m=1, n \geq 1$.

\begin{tabular}{|c|c|c|c|}
\hline$\left\{r_{u}, r_{v}\right\}$ & $f=\left|R E_{u, v}\right|$ & $\begin{array}{l}r_{u} \\
+r_{v}\end{array}$ & $f \cdot\left(r_{u}+r_{v}\right)$ \\
\hline$\{3,2\}$ & 2 & 5 & 10 \\
\hline$\{2,2\}$ & $5 n+2$ & 4 & $20 n+4$ \\
\hline$\{2,1\}$ & $6 n-6$ & 3 & $18 n-18$ \\
\hline$\{1,1\}$ & $\begin{array}{l}15 m n-15 n \\
-2 m+2\end{array}$ & 2 & $\begin{array}{l}30 m n-30 n \\
-4 m+4\end{array}$ \\
\hline
\end{tabular}

Adding entries of last column, we get $R_{1}(G)$ for $m>1$, $n \geq 1$.

\begin{tabular}{|c|c|c|c|}
\hline$\left\{r_{u}, r_{v}\right\}$ & $f=\left|R E_{u, v}\right|$ & $\begin{array}{c}r_{u} \\
+r_{v}\end{array}$ & $f \cdot\left(r_{u}+r_{v}\right)$ \\
\hline$\{3,2\}$ & 2 & 5 & 10 \\
\hline$\{2,2\}$ & $2 n+2$ & 4 & $8 n+8$ \\
\hline$\{2,1\}$ & $\begin{array}{l}12 n+8 m \\
-14\end{array}$ & 3 & $36 n+24 m-42$ \\
\hline$\{1,1\}$ & $\begin{array}{c}15 m n-18 n \\
-10 m+10\end{array}$ & 2 & $\begin{array}{l}30 m n-36 n \\
-20 m+20\end{array}$ \\
\hline
\end{tabular}

Revan first polynomial $R_{1}(G, x)$ for $m=1, n \geq 1$ is

$$
\sum_{u v \in E(G)} x^{\left(r_{u}+r_{v}\right)}=2 x^{5}+(5 n+2) x^{4}+(6 n-6) x^{3}+
$$

$(15 m n-15 n-2 m+2) x^{2}$.

Revan first polynomial for $m>1, n \geq 1$ is

$$
R_{1}(G, x)=2 x^{5}+(2 n+2) x^{4}+(12 n+8 m-14) x^{3}+
$$

$(15 m n-18 n-10 m+10) x^{2}$.

Adding entries of last column, we get

$$
R_{2}(G) \text { for } m=1, n \geq 1
$$

\begin{tabular}{|c|c|c|c|}
\hline$\left\{r_{u}, r_{v}\right\}$ & $f=\left|R E_{u, v}\right|$ & $r_{u} \cdot r_{v}$ & $f \cdot\left(r_{u} \cdot r_{v}\right)$ \\
\hline$\{3,2\}$ & 2 & 6 & 12 \\
\hline$\{2,2\}$ & $5 n+2$ & 4 & $20 n+8$ \\
\hline$\{2,1\}$ & $6 n-6$ & 2 & $12 n-12$ \\
\hline$\{1,1\}$ & $\begin{array}{l}15 m n-15 n \\
-2 m+2\end{array}$ & 1 & $\begin{array}{l}15 m n-15 n \\
-2 m+2\end{array}$ \\
\hline
\end{tabular}


Adding entries of last column, we get $R_{2}(G)$ for $m>1$, $n \geq 1$.

\begin{tabular}{|c|c|c|c|}
\hline$\left\{r_{u}, r_{v}\right\}$ & $f=\left|R E_{u, v}\right|$ & $r_{u} \cdot r_{v}$ & $f \cdot\left(r_{u} \cdot r_{v}\right)$ \\
\hline$\{3,2\}$ & 2 & 6 & 12 \\
\hline$\{2,2\}$ & $2 n+2$ & 4 & $8 n+8 m$ \\
\hline$\{2,1\}$ & $\begin{array}{l}12 n+8 m \\
-14\end{array}$ & 2 & $24 n+16 m-28$ \\
\hline$\{1,1\}$ & $\begin{array}{l}15 m n-18 n \\
-10 m+10\end{array}$ & 1 & $\begin{array}{l}15 m n-18 n \\
-10 m+10\end{array}$ \\
\hline
\end{tabular}

Revan second polynomial $R_{2}(G, x)$ for $m=1, n \geq 1$

$\sum_{u v \in E(G)} x^{\left(r_{u} \cdot r_{v}\right)}=2 x^{6}+(2 n+2) x^{4}+(12 n+8 m-$

14) $x^{2}+(15 m n-18 n-10 m+10) x$.

Revan second polynomial for $m>1, n \geq 1$ is

$R_{2}(G, x)=2 x^{6}+(5 n+2) x^{4}+(6 n-6) x^{2}+$ $(15 m n-15 n-2 m+2) x$.

Adding entries of last column, we get

$R_{3}(G)$ for $m=1, n \geq 1$.

\begin{tabular}{|l|c|c|c|}
\hline$\left\{r_{u}, r_{v}\right\}$ & $f=\left|R E_{u, v}\right|$ & $\begin{array}{c}\mid r_{u} \\
-r_{v} \mid\end{array}$ & $f \cdot\left|r_{u}-r_{v}\right|$ \\
\hline$\{3,2\}$ & 2 & 1 & 2 \\
\hline$\{2,2\}$ & $5 n+2$ & 0 & 0 \\
\hline$\{2,1\}$ & $6 n-6$ & 1 & $6 n-6$ \\
\hline$\{1,1\}$ & $\begin{array}{l}15 m n-15 n \\
-2 m+2\end{array}$ & 0 & 0 \\
\hline
\end{tabular}

Adding entries of last column, we get $R_{3}(G)$ for $n>1$, $m \geq 1$.

\begin{tabular}{|c|c|c|c|}
\hline$\left\{r_{u}, r_{v}\right\}$ & $f=\left|R E_{u, v}\right|$ & $\begin{array}{c}\mid r_{u} \\
-r_{v} \mid\end{array}$ & $f \cdot\left|r_{u}-r_{v}\right|$ \\
\hline$\{3,2\}$ & 2 & 1 & 2 \\
\hline$\{2,2\}$ & $2 n+2$ & 0 & 0 \\
\hline$\{2,1\}$ & $\begin{array}{c}12 n+8 m \\
-14\end{array}$ & 1 & $12 n+8 m-14$ \\
\hline$\{1,1\}$ & $\begin{array}{c}15 m n-18 n \\
-10 m+10\end{array}$ & 0 & 0 \\
\hline
\end{tabular}

Revan third polynomial is $R_{3}(G, x)$ for $m=1, n \geq 1$

$\sum_{u v \in E(G)} x^{\left|r_{u}-r_{v}\right|}=(6 n-4) x+(15 m n-10 n-2 m+4)$. Revan third polynomial for $m>1, n \geq 1$ is

$R_{3}(G, x)=(12 n+8 m-12) x+(15 m n-16 n-10 m+$ 12).

\section{ANALYSIS}

- $\quad$ Comparison of Revan first index $R_{1}(G)$, Revan second index $R_{2}(G)$ and Revan third index $R_{3}(G)$ for $S_{2} C_{3}-I[n, m]$. Blue color sheet represents Revan first index, purple color sheet represents Revan second index and green color sheet represents Revan third index.

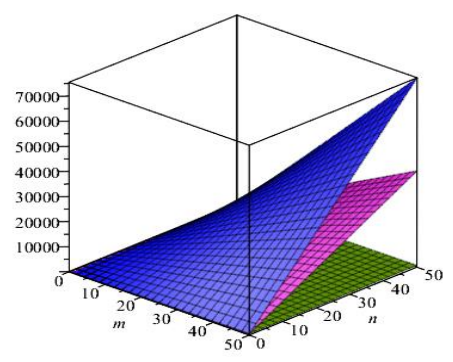

- Comparison of Revan first index $R_{1}(G)$, Revan second index $R_{2}(G)$ and Revan third index $R_{3}(G)$ of $\mathrm{Si}_{2} \mathrm{C}_{3}-I I[n, m]$. Black color sheet represents Revan first index, yellow color sheet represents Revan second index and red color sheet represents Revan third index.

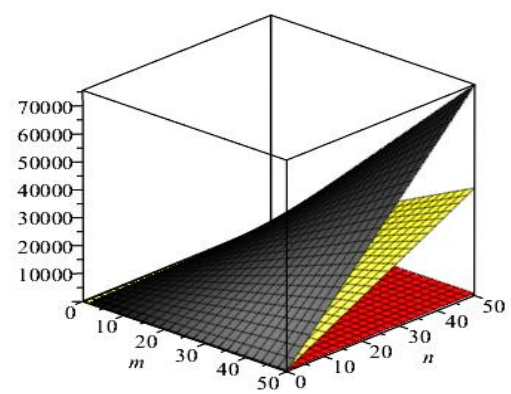

Comparison of Revan first index $R_{1}(G)$, Revan second index $R_{2}(G)$ and Revan third index $R_{3}(G)$ of $\mathrm{Si}_{2} \mathrm{C}_{3}-I I I[n, m]$. Sky color sheet represents Revan first index, yellow color sheet represents Revan second index and purple color sheet represents Revan third index.

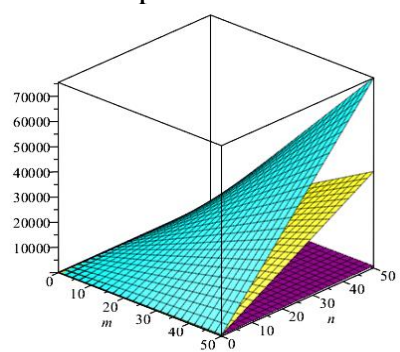

Comparison of Revan first index $R_{1}(G)$, Revan second index $R_{2}(G)$ and Revan third index $R_{3}(G)$ of $\mathrm{SiC}_{3}-$ $I[n, m]$. Yellow color sheet represents Revan first index, blue color sheet represents Revan second index and red color sheet represents Revan third index.

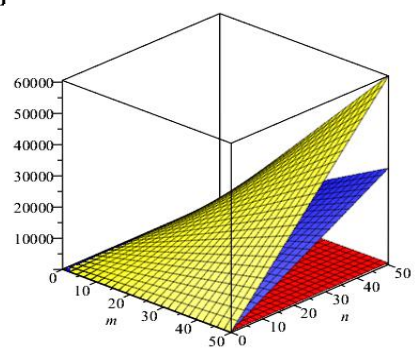

- Comparison of Revan first index $R_{1}(G)$, Revan second index $R_{2}(G)$ and Revan third index $R_{3}(G)$ of $\mathrm{SiC}_{3}-$ $I I[n, m]$. White color sheet represents Revan first index, sea green color sheet represents Revan second index and yellow color sheet represents Revan third index. 


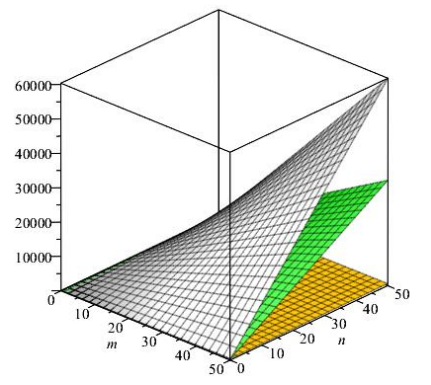

- $\quad$ Comparison of Revan first index $R_{1}(G)$, Revan second index $R_{2}(G)$ and Revan third index $R_{3}(G)$ of $\mathrm{SiC}_{3}-$ $I I I[n, m]$. Green color sheet represents Revan first index, sky blue color sheet represents Revan second index and yellow color sheet represents Revan third index.

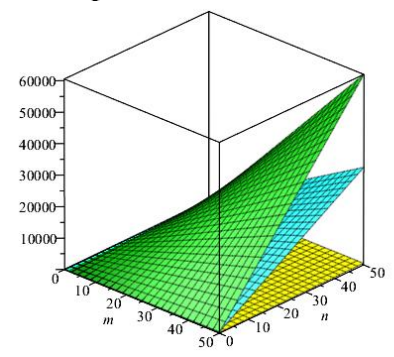

- $\quad$ Comparison of Revan first index $R_{1}(G)$, Revan second index $R_{2}(G)$ and Revan third index $R_{3}(G)$ of $\mathrm{SiC}_{4}-$ $I[n, m]$. Yellow color sheet represents Revan first index, blue color sheet represents Revan second index and sky blue color sheet represents Revan third index.

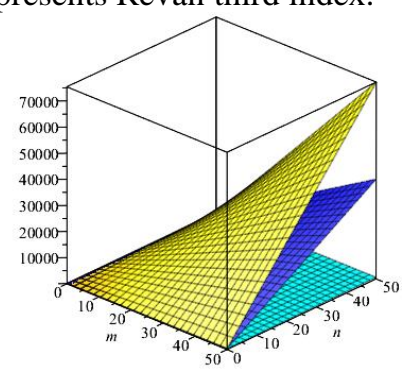

- $\quad$ Comparison of Revan first index $R_{1}(G)$, Revan second index $R_{2}(G)$ and Revan third index $R_{3}(G)$ of $\mathrm{SiC}_{4}-$ $I[n, m]$. Red color sheet represents Revan first index, sky blue color sheet represents Revan second index and purple color sheet represents Revan third index.

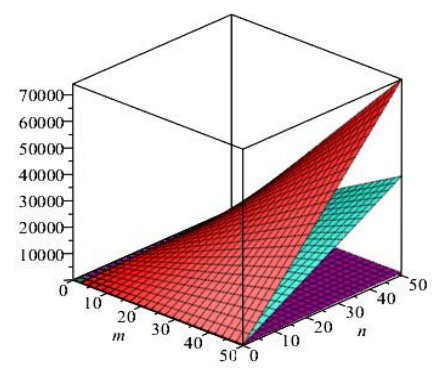

\section{REFERENCES}

[1] J. Devillers, A. T. Balaban, Topological indices and related descriptors in QSAR and QSPR. Gordon and Breach, Amsterdam, 1999.

[2] M. Karelson, Molecular descriptors in $Q S A R / Q S P R$. Wiley-Inter science, New York, 2000.

[3] M. Kamran, M. K. Siddiqui, M. Naeem, M. A. Iqbal, On topological properties of symmetric chemical structures. Symmetry 2018, 10(5), 173 April (2018).

[4] V. R. Kulli, 2012. College Graph Theory, Vishwa International Publications,Gulbarga, India.

[5] V. R. Kulli, Revan indices of oxide and honeycomb networks, International Journal of Mathematics and its Applications, 5(4-E) (2017) 663-667.

[6] V. R. Kulli. Revan indices and their polynomials of certain Rhombus networks. ReserachGate article, International Journal of Current Research in Life Sciences. Vol.07, No. 05, pp. 2110-2116, May, 2018.

[7] V. R. Kulli. Hyper-Revan indices and their polynomials of silicate networks. International Journal of Current Research in Science and Technology.Volume 4, Issue3 (2018), 17-21.

[8] V. R. Kulli, On $K$ edge index of some nanostructures, Journal of Computer and Mathematical Sciences, 7(7), (2016) 373-378.

[9] V. R. Kulli, On the sum connectivity Gourava index, International Journal of Mathematical Archive, 8(7)(2017) 211-217.

[10] Y. C. Kwun, A. R. Virk, W. Nazeer, M. A. Rehman, S. M. Kang. On the multiplicative degree based topological indices of siliconcarbon $\mathrm{Si}_{2} C_{3}-I[p, q]$ and $\mathrm{Si}_{2} C_{3}-I I[p, q]$. Symmetry 2018, 10, 320 .

[11] H. Wiener, Structural determination of paraffin boiling points. J. Amer. Chem. Soc. 69 (1947), 17-20. 